\title{
A 2R Mamyshev Regeneration Architecture Based on a Three-Fiber Arrangement
}

\author{
L. Provost, C. Finot, P. Petropoulos, D.J. Richardson
}

\begin{abstract}
The benefits of using a multi-segmented arrangement of optical fibers for Self-Phase Modulation-based $2 R$ optical regeneration are demonstrated both theoretically and experimentally. Significant improvements in terms of the performance and practicality are achieved.
\end{abstract}

Index Terms - All-optical regeneration, Nonlinear optical devices, Optical Kerr effect, Self-phase modulation.

\section{INTRODUCTION}

$\mathrm{W}$ ith the development of long haul optical telecommunication systems working at high repetition rates $(40 \mathrm{Gbit} / \mathrm{s}$ and beyond), performing alloptical regeneration of the signal has become of great interest to combat the various cumulative impairments that occur during signal propagation and that include: noise accumulation due to amplified spontaneous emission of the optical amplifiers, chromatic dispersion as well as intrachannel nonlinear effects. As a consequence, in order to improve the transmission distance, it is generally necessary to limit the amplitude jitter of the pulses and to enhance the extinction ratio (ER) of the signal. To simultaneously achieve such operations at high bit rates, different alloptical approaches based on instantaneous nonlinear effects in optical fibers have been proposed and implemented in devices, such as non-linear optical loop mirrors [1] and wavelength-converters based on either cross-phase modulation [2] or four-wave mixing [3].

Amongst these fiber based regeneration methods, the 2Rscheme proposed by Mamyshev in 1988 [4], which relies on Self-Phase Modulation (SPM) induced spectral broadening in a normally dispersive fiber and offset filtering, is particularly attractive due to its relative simplicity and potential for low-cost implementation. As a consequence numerous works demonstrating ER improvement, amplitude jitter reduction under both single [5-15] and multi-channel operation have been previously reported [16-18]. Several aspects of its operation can be physically linked to the interaction between the Kerr nonlinearity and dispersive

Manuscript received September 15, 2009.

This work was partially supported by the European Union FP7 NoE BONE. L. Provost, P. Petropoulos and D.J. Richardson are with the Optoelectronics Research Centre, University of Southampton, SO17 1BJ, United-Kingdom

C. Finot is with the laboratoire Interdisciplinaire CARNOT de Bourgogne, UMR 5209 CNRS - Université de Bourgogne, 9 Av. A. Savary, BP 47870, 21078 Dijon Cedex, France (e-mail: christophe.finot@u-bourgogne.fr). effects and it has been shown that the value of the fiber dispersion strongly influences the performance of the device [5-7]. In particular, it has been demonstrated that the realization of a plateau for the ' 1 ' symbols in the power transfer function can be more optimally realized if the regenerator operates close to the wave-breaking condition [19-21], which requires a particular balance between integrated dispersion and nonlinearity [5].

Recently, the possibility to efficiently manipulate such wave-breaking conditions using an assembly of fibers with different dispersive/nonlinear properties [22] or using a tapered fiber [23] was experimentally demonstrated leading us to question if such an approach might be employed in the context of Mamyshev regeneration.

In this article, we show that the concept of dispersion and nonlinearity management can indeed be beneficial for optical regeneration. Specifically, we demonstrate that inserting a small segment of dispersion compensating fiber (DCF) (viewed as a lumped dispersive element), between two highly nonlinear fibers (HNLFs) (representing lumped nonlinear elements) results in a suitable Mamyshev regenerator solution offering higher design flexibility as compared to a single-fiber case. We support the proposed concept both theoretically and experimentally highlighting that the amplitude noise compression properties of the basic approach are advantageously preserved. Most interestingly, the scheme can be operated at different signal bitrates simply by changing the properties of the lumped dispersive element which thereby very usefully relaxes the constraints and specifications placed on the HNLF [24].

\section{PROPOSED SET-UP AND NUMERICAL SIMULATIONS}

A. Design issues in conventional Mamyshev Regenerator A schematic of the conventional Mamyshev regenerator is shown in Fig. 1 [4]. An Erbium doped fiber amplifier (EDFA) boosts the incoming signal to the power $\left(\mathrm{P}_{\mathrm{m}}\right)$ required for optimal peak power equalization of the 'one' symbols. The SPM-induced spectral broadening is generated in the normally dispersive fiber (NDF) which has a chromatic dispersion $\mathrm{D}$, a nonlinear coefficient $\gamma$, and linear losses $\alpha$. At the fiber output, a Gaussian optical bandpass filter (OBPF) with a FWHM spectral width $\delta$, (defined such that the pulse width at the output is the same as that at the input of the system), is spectrally offset by an amount $\Delta \lambda$ with respect to the input signal carrier wavelength $\lambda_{0}$ and is used to carve into the broadened 
spectrum - thereby acting as a pulse reshaper.

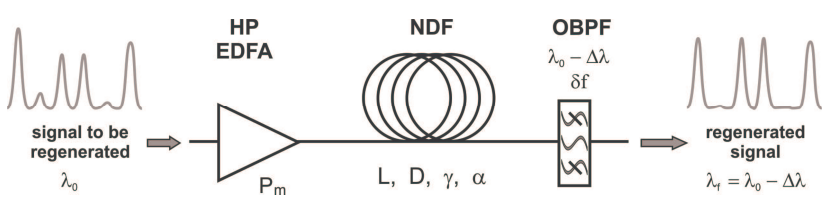

Fig. 1: Conventional Mamyshev regenerator set-up.

The performance of a $2 \mathrm{R}$ regenerator can primarily be assessed by considering its transfer function (TF) which relates the output to input pulse energies. Optimum power equalization occurs when the TF regime exhibits a plateau for the 'one' input level over a range of input pulse energies. As shown in [5, 8], such a regime can be obtained when precise relationships between the input pulse and NDF parameters are satisfied. Table 1 shows some of the physical properties of five different types of NDF, namely a Dispersion Shifted Fiber (DSF), two HNLFs, a DCF and a highly nonlinear bismuth oxide fiber (BOF). The incoming signal considered is a PRBS return-to-zero pulse train with a data rate $\mathrm{R}=40 \mathrm{~Gb} / \mathrm{s}$ and $25 \%$ duty cycle. The frequency detuning $\Delta \lambda$ is (arbitrarily) set to $2.5 \times \mathrm{R}$ (i.e. $100 \mathrm{GHz}$ ). Then the associated lengths $L$ and average input powers $P_{m}$ required to achieve a suitable $\mathrm{TF}$ are also presented in Table 1.

From Table 1, it is clear that a trade-off between fiber length/dispersion and the required power $\mathrm{P}_{\mathrm{m}}$ budget has to be found. Achieving compact devices therefore dictates the use of fiber with high dispersion values, such as DSF or DCF. Unfortunately, the high average powers required for regenerators based on these types of fibers render them impractical. Consequently, one has to consider the use of highly nonlinear fibers. However, as those fibers are usually designed to have low-dispersion values, several kilometers of HNLFs are needed. Producing such lengths of HNLF with suitably accurate control of the dispersion is both technically challenging and likely to be very expensive. Approaches to reduce the length requirements and specifications on such fibers for use in Mamyshev regenerators are thus highly desirable. Devices based on photonic crystal fiber structures or non-silica glasses such as bismuth oxide [9] or chalcogenide [6] present a very attractive solution and could overcome those limitations, but to date, they are not widely available and they still suffer from high losses.

TABLE 1: FIBER PROPERTIES AND REQUIREMENTS FOR OPTIMAL REGENERATION FOR $25 \%$ RZ SIGNALS AT $40 \mathrm{~GB} / \mathrm{S} \quad(\Delta \lambda=2.5 \mathrm{XR})$

\begin{tabular}{|c|c|c|c|c|c|}
\hline $\begin{array}{l}\text { Fiber } \\
\text { Type }\end{array}$ & $\begin{array}{c}\text { D } \\
\mathrm{ps} / \mathrm{nm} / \mathrm{km}\end{array}$ & $\begin{array}{c}\gamma \\
/ \mathrm{W} / \mathrm{km}\end{array}$ & $\begin{array}{c}\alpha \\
\mathrm{dB} / \mathrm{km}\end{array}$ & $\begin{array}{l}L \\
\mathrm{~m}\end{array}$ & $\begin{array}{c}P_{m} \\
\mathrm{dBm}\end{array}$ \\
\hline DSF & -7 & 1.8 & 0.2 & 402 & 32.9 \\
\hline HNLF 1 & -0.87 & 20 & 1.0 & 3,602 & 14.8 \\
\hline HNLF 2 & -0.64 & 20 & 0.5 & 4,712 & 13.0 \\
\hline DCF & -120 & 5.0 & 0.6 & 23.4 & 40.8 \\
\hline BOF & -260 & 1100 & 900 & 15 & 24.0 \\
\hline
\end{tabular}

\section{B. Pulse dynamics in the proposed solution}

Our proposed solution is presented in Fig. 2. It consists of replacing the single fiber with a set of three fibers showing almost either purely dispersive or nonlinear behavior. A greater control of the nonlinear and dispersive signal evolution through the regenerator is then possible. The proposed arrangement relies on a separation of the dispersive and nonlinear contributions to the pulse evolution in a normally dispersive regime.

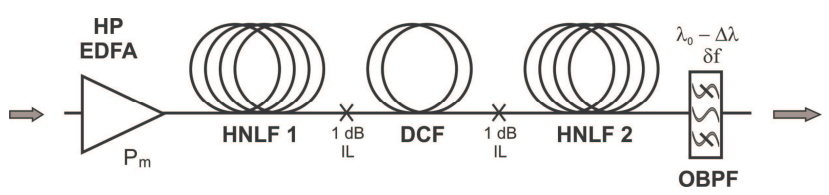

Fig. 2: Proposed set-up based on a three-fiber segment arrangement.

In order to assess the validity of this new architecture, we have simulated the propagation of a single chirp-free Gaussian pulse in the proposed regenerator [5]. The longitudinal evolution of the complex electric field envelope $\psi(z, T)$ in an optical fiber can be modeled in terms of the well-known nonlinear Schrödinger equation :

$$
i \frac{\partial \psi}{\partial z}=\frac{\beta_{2}}{2} \frac{\partial^{2} \psi}{\partial T^{2}}-\gamma|\psi|^{2} \psi-i \frac{\alpha}{2} \psi,
$$

with $\beta_{2}$ the second order dispersion of the fiber. As we have considered dispersion flattened fibers or fibers with a high $\beta_{3} / \beta_{2}$ ratio, we have neglected the influence of the third order dispersion $\beta_{3}$ for the sake of simplicity. $\gamma$ is the effective Kerr nonlinearity coefficient. The distributed linear fiber losses are defined by $\alpha$ and the insertion losses (IL) associated with the DCF have also been taken into account

A 500 m-long HNLF (HNLF 1 in Table 1) is first used to generate SPM thereby imposing a non-monotonic frequency chirp across the pulse as seen in the results of Fig. 3(a) which leads to significant spectral broadening (Fig. 3(b)). The low dispersion of HNLF1 ensures that the pulse temporal intensity profile is maintained throughout this fiber. Note that an anomalously dispersive HNLF could in fact also have been used as HNLF1 without any change in performance which is in contrast to the standard Mamyshev setup where the fiber used has to be normally dispersive.
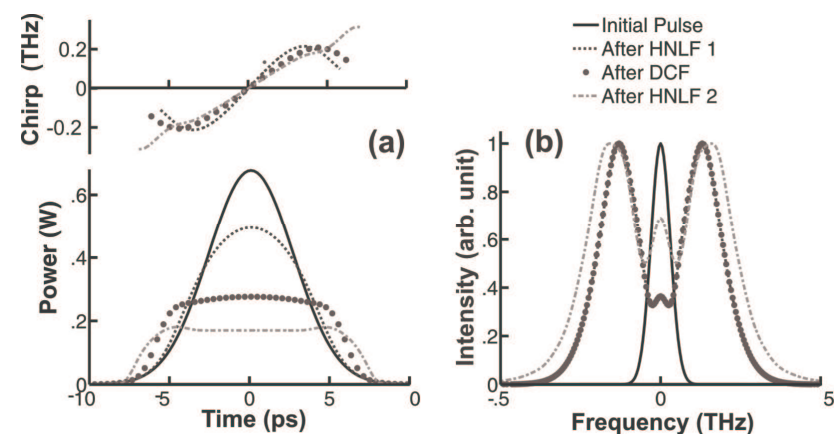

Fig. 3: Numerical evolution of (a) the temporal intensity (bottom) and chirp (top) profiles and (b) of the optical spectrum for an initial pulse energy of $4.5 \mathrm{pJ}$ at various points within the 3-segment configuration. 
The second section is a 6-m length of DCF which provides a large amount of normal dispersion. A total DCF IL of $\sim 2 \mathrm{~dB}$ was assumed. The temporal intensity of the pulse profile is clearly impacted with a flattening over most of the pulse. The spectrum is however unaffected, confirming the quasi-linear nature of this propagation stage.

The last fiber is another 500 m-long HNLF (HNLF 2). Nonlinear propagation of the pulses shaped within the DCF in HNLF 2 results in further spectral enrichment, as can be seen in Fig. 3(b) as well as a linearization of the chirp of the pulse.

Note that the goal of this fiber arrangement is intentionally opposite to that proposed in $[22,25]$ where an increase in the ratio of the strength of the non-linear and dispersive effects is used to avoid wave-breaking. Let us additionally point out that our approach differs from the fiber arrangement proposed by Vasilyev et al. [16, 18] which is targeted at reducing inter-channel crosstalk effects under multichannel operation and where the regenerator comprises numerous sections of fiber with alternating signs of dispersion to control pulse-to-pulse interactions. Moreover, contrary to step-like or comb-like [25-27] architectures where tens of segments are required to mimic an adiabatic evolution of the dispersion profile, our set-up is physically based on just three normally dispersive fiber segments and no significant improvement is expected from the use of a larger number of segments.
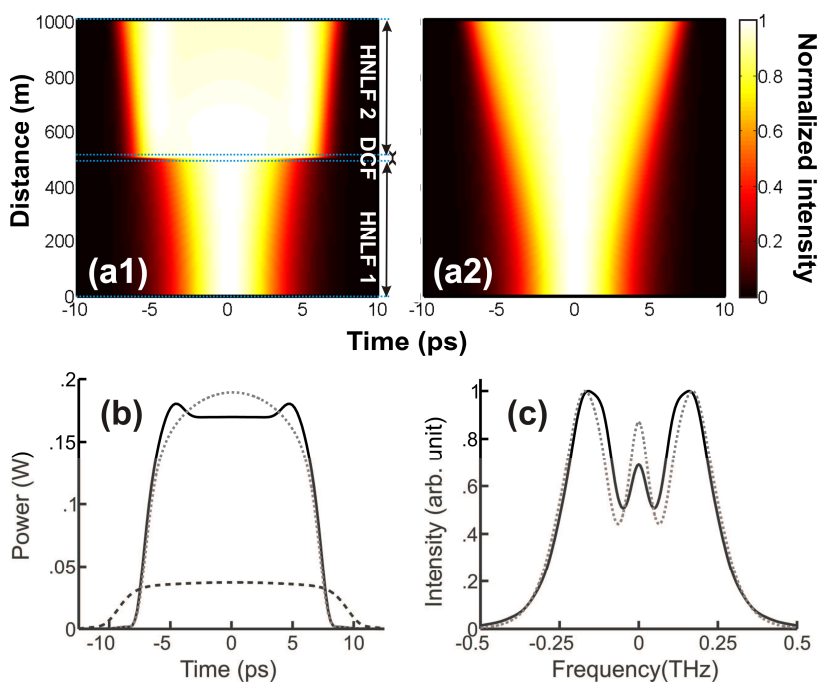

Fig. 4: (a) Longitudinal evolution of the temporal intensity profile in the multi-segment architecture (a1) and in an imaginary fiber having similar average parameters (a2). The temporal (b) and spectral (c) intensity profiles at the output for both configurations are compared (solid black lines and dotted grey lines for the multi-segment architecture and the single fiber respectively). The intensity profile obtained after propagation in $3.7 \mathrm{~km}$ of HNLF 1 is also plotted (black dashed line).

In order to get further insight into the pulse dynamics undergone in our fiber arrangement, we have plotted in Fig. 4 (a1) the longitudinal temporal evolution of a Gaussian pulse. The three previously mentioned stages are clearly visible in this plot. It is also of interest to compare this evolution with that experienced in a single uniform length of a hypothetical fiber having physical parameters (dispersion, non-linearity, losses) that correspond to the weighted average values of the three segments and a length corresponding to the total length of the arrangement. In such an imaginary fiber $(\mathrm{D}=-1.46 \mathrm{ps} / \mathrm{km} / \mathrm{nm}, \gamma=19.9 / \mathrm{W} / \mathrm{km}, \alpha$ $=2.74 \mathrm{~dB} / \mathrm{km}$ ), the temporal evolution is continuous (Fig. 4 (a2)) and the output pulses obtained (Fig. 4 b-c) clearly differ from those obtained in the three-segment arrangement. More precisely, the parabolic-like pulse obtained in the single fiber is characteristic of a pre- wavebreaking situation $[20,21]$ whereas the flattening of the top of the pulse is an indication of post- wave-breaking evolution. Such differences confirm that the DCF cannot be considered as a simple localized feature affecting only the average parameters of the line.

\section{Impact of the intermediate DCF fiber}

The detailed shaping of the output spectrum is controlled by the amount of dispersion provided by the DCF. Indeed, increasing the DCF length contributes to increased broadening of the temporal intensity profile (Fig. 5a). As the SPM induced in the third segment depends on the temporal intensity gradient of the pulses, the DCF length will then also directly impact the optical spectrum (Fig. 5(c)) and we can observe significant spectral reshaping around the frequency of $100 \mathrm{GHz}$ (the central wavelength of the offset spectral filter).
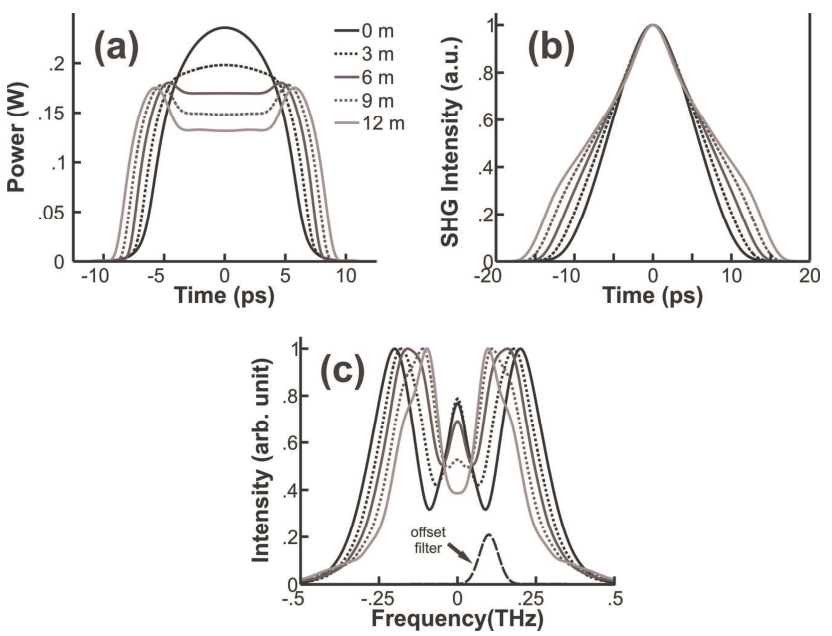

Fig. 5: Influence of the intermediate DCF length on the temporal (a) and spectral (c) intensity profiles obtained before spectral filtering. Fig. (b) is the intensity autocorrelation associated with the temporal profiles of Fig. 5(a).

These spectral changes are also reflected in the overall TF obtained after the offset filtering. As illustrated in Fig. 6(a), the exact $\mathrm{TF}$ shape can be tuned and the TF flatness can be substantially modified by varying the DCF length. A $6 \mathrm{~m}$-long DCF is optimal with the current choice of fibers. Note that in the absence of any DCF ( $0 \mathrm{~m}$ case), 
the ripple in the TF is prohibitive. These results clearly highlight the potential benefits of our proposed scheme where only $1,006 \mathrm{~m}$ of fiber is required. This has to be compared to the $3-\mathrm{km}$ long HNLF required for the single stage regenerator (see Table 1).

These results can also be compared to the TF obtained with the ideal imaginary fiber introduced in the previous section and once again, clear differences can be noticed. Furthermore, the order in which the fiber segments are arranged is particularly crucial: Fig. 6(b) highlights that suitable tuning of the TF is only observed when the DCF is placed between the two HNLFs, which is consistent with our physical explanation of the process and which definitively confirms the non-linear behavior of our fiber arrangement.
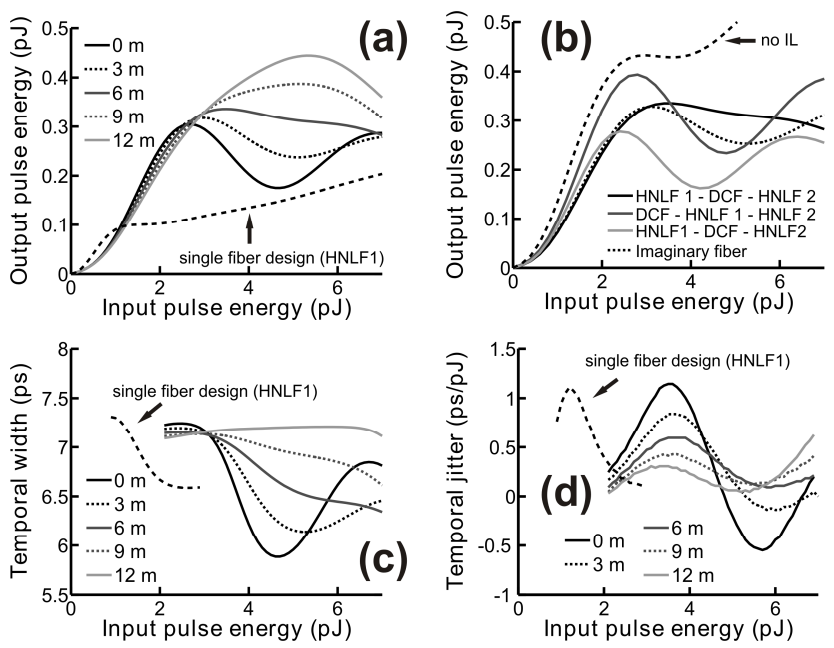

Fig. 6: (a) TFs obtained for various intermediate DCF lengths compared with the TF based on a $3.6 \mathrm{~km}$ HNLF 1. (b) TF obtained with a 6-m DCF placed between HNLF1 and HNLF2 compared with other orders of fiber arrangement and with the TF resulting from a single uniform hypothetical fibre of the same average parameters. (c) FWHM temporal duration of the pulses emerging from the regenerator (the same convention is used as in Fig. 6(a)) (d) Temporal pulse energy-dependant jitter (defined as the derivative of the output delay of the pulse with respect to energy ) for various intermediate lengths of DCF (same convention as Fig. (a)).

Some additional benefits of the multi-segment architecture over the single fiber design are worth mentioning. Due to the fundamental modification of the interplay between nonlinearity and dispersion, we observe that the relative spectral broadening ratio between pulses of high and low intensities is increased as compared to the single fiber design. This demonstrates that the noise rejection capability is intrinsically increased for the multisegment design. Considering the single fiber design using HNLF 1 (Table 1) and an input extinction ratio ER of 10dB, we obtained an output ER of $12.5 \mathrm{~dB}$ for the single fiber case, which is to be compared to $16 \mathrm{~dB}$ for the multisegment setup (6 m-long DCF). Furthermore, the multisegment scheme is slightly more power efficient. Specifically, the energy yield (defined as the ratio between output and input energy) is $\sim 8.1 \%$ for the multi-segment segment, as compared to $5.6 \%$ obtained for the single fiber design based on HNLF 1 . By eliminating the $2 \mathrm{~dB}$ insertion losses of the DCF, the yield could be as high as $12.5 \%$.

The DCF segment also impacts the temporal duration of the pulse obtained after spectral filtering (Fig. 6(c)) as well as the timing jitter inherent to the regeneration process (Fig. 6(d) : the output delay of the regenerated pulses varies according to the incoming pulse energy. The time derivative of this delay with respect to the input energy provides a quantitative estimate of the resulting additional timing jitter) $[5,8,10]$. The performance of the multi-segmented architecture on these two factors for a $6 \mathrm{~m}$ length is found to be quite close to that of a single fiber architecture. However, our study reveals that for longer segments of intermediate DCF, a dramatic reduction in the output pulse duration fluctuations and of the detrimental powerdependent timing delays can be achieved. Therefore, a trade-off between TF flatness and the temporal properties of the regenerated pulses should ideally be found. Such a detailed study is far beyond the scope of the present paper and we only focus here on the TF improvement.

Finally, the temporal duration of the pulses before filtering is more limited in our multi-segment approach. For a configuration based on a single HNLF (HNLF1), the pulse broadens up to a FWHM duration of $18 \mathrm{ps,} \mathrm{which} \mathrm{is} \mathrm{to} \mathrm{be}$ compared with 13.5 ps obtained in the multi-segmented architecture (see Fig. 4(b)). This means that our approach is likely to offer better resistance to intra-channel crosstalk (iFWM, iXPM) along the regenerator and to avoid prohibitive pulse patterning effects [5, 10]. This advantageous feature will be even more important for lower duty-cycle signals such as RZ-33\%.

The various conclusions drawn in this section were obtained by studying a regenerator based on two $500-\mathrm{m}$ HNLFs combined with a frequency detuning $\Delta \lambda$ of the output filter which was arbitrarily fixed to $2.5 \times \mathrm{R}$. Additional numerical simulations with other frequency detunings have revealed that the key advantages of the multi-segmented configuration were maintained over a wide range of $\Delta \lambda$ (as long as the concatenation of the two HNLFs leads to a non-monotonous transfer function). Similarly, the length of the two segments of HNLFs has not been found to be a critical parameter: for a wide range of HNLF lengths, we have managed to find a suitable DCF segment.

\section{Bidirectional use of the regenerator}

It is to be appreciated that our scheme is intentionally designed to be symmetric, so that it is fully compatible with both multi-channel and wavelength-conversion free device implementations which impose bidirectional propagation through the regenerator $[11,17]$. 

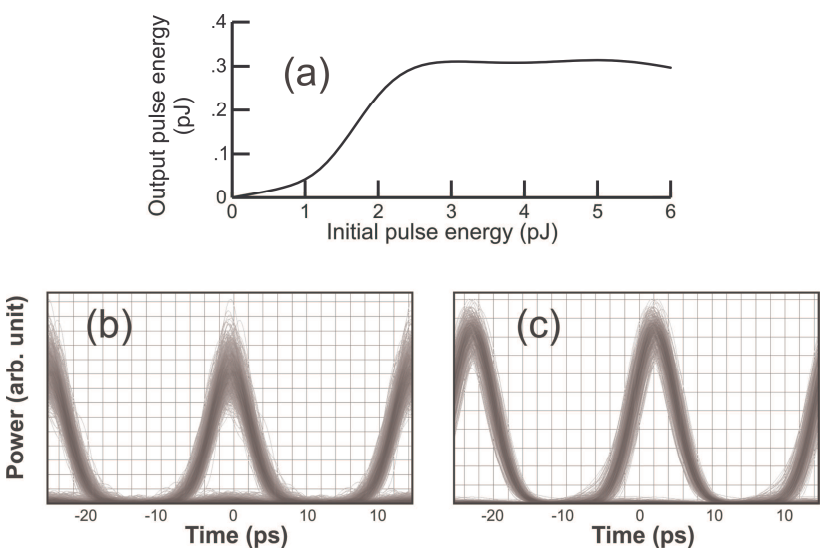

Fig. 7: (a) TF obtained for a bidirectional use. at the regenerator input (b) and output (c)

(b) Optical eye diagram

The TF resulting from bidirectional use is plotted in Fig. 7(a). An intermediate amplifier with $9 \mathrm{~dB}$ gain enables compensation of losses in the first regeneration stage. A remarkable plateau ranging from 2 to $6 \mathrm{pJ}$ is obtained, enabling a highly effective optical limiting capability.

In order to test the ability of the device to efficiently regenerate a distorted signal, we have numerically injected a degraded $40 \mathrm{~Gb} / \mathrm{s}$ signal (obtained after propagation in a realistic transmission line made of segments of single mode fibers compensated by DCF, see ref [10] for additional details). Whereas previous discussions relied on the study of a single Gaussian pulse evolving in the regenerator [5], we consider here a pseudo-random bit sequence of 4096 pulses so that potential data patterning effects are taken into account as well as the temporal fluctuations of the incoming pulse duration.

The input eye diagram is plotted in Fig. 7(b) and can be compared with the regenerated eye diagram plot in Fig. 7(c). For optimized input average powers and intermediate amplifier gains, a clear improvement is visible, both on the marks and spaces of the signal: fluctuations in the zero bit slots have been significantly reduced as well as the amplitude fluctuations of the pulse. The resulting eyeopening is confirmed by the improvement in the Q-factor [28] evaluated after electrical detection (the Q-factor before regeneration is 11.3 and this is improved after regeneration to 16.5$)$.

\section{EXPERIMENTAL SET-UP AND RESULTS}

In order to validate the principle of our approach we first experimentally implemented the technique with pulses of 6.4 ps duration (typical of $25 \% \mathrm{RZ} 40 \mathrm{~Gb} / \mathrm{s}$ systems). These pulses were generated by a Gain-Switched DFB laser diode operating at a repetition rate of $10 \mathrm{GHz}$. Autocorrelation measurements (Fig. 8(a)) confirm the previously described impact of the different segments on the temporal shape of the pulse: whereas the propagation in the first segment does not influence the temporal intensity profile, a significant temporal broadening is experienced in the DCF segment and finally the evolution in the last HNLF segment is mainly nonlinear. The pulses recovered after filtering are very similar to those of the incoming pulses.

The influence of the intermediate length of DCF on the properties of the pulse obtained before spectral slicing is depicted in Figs. 8(b-c). The trends observed in the (Second Harmonic Generation (SHG)) autocorrelation signals (Fig.8(b)) are in a clear agreement with the numerical modeling (Fig. 5(b)) and the experimental data exhibits both a temporal broadening as well as a marked change in the temporal shape with increasing DCF length. Spectral reshaping (Fig.8(c)) is clearly observed for negative frequency filter offsets, whereas the reshaping effect is less distinguishable for positive frequency offsets with more pronounced spectral ripples. We attribute this observation to the presence of an asymmetric chirp on the incoming pulse profile.
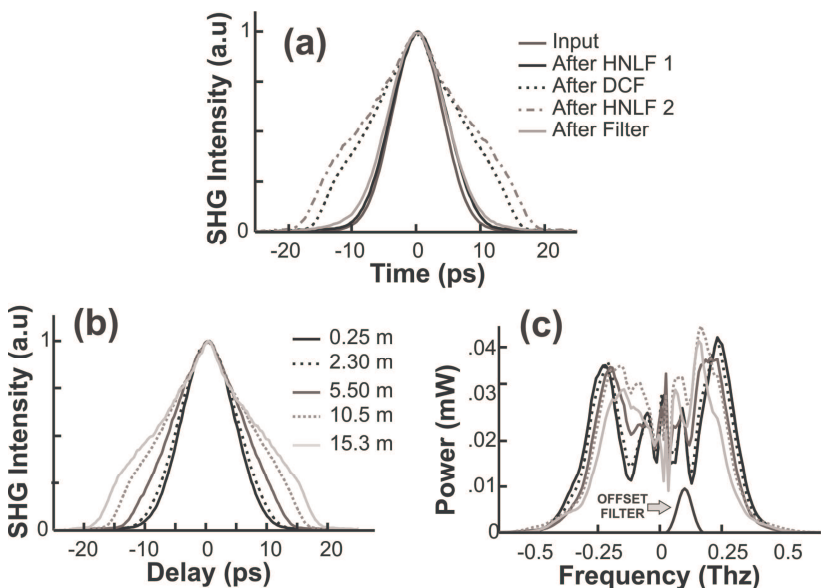

Fig. 8: Experimental results with a $10 \mathrm{GHz}, 6.4$ ps input pulse train: (a) Autocorrelation traces recorded at different output positions for a $10.5 \mathrm{~m}$ long DCF. (b-c) Influence of the DCF length for an input average power of $110 \mathrm{~mW}$ : (b) autocorrelation traces after the DCF before optical filtering and (c) optical associated spectra.

The corresponding TFs obtained after offset filtering by $100 \mathrm{GHz}(\delta \mathrm{f}=66 \mathrm{GHz})$ are shown in Fig. 9(a2). A reshaping of the TF is obtained for various lengths of DCF. Just $10.5 \mathrm{~m}$ are required to obtain a flat plateau. These results are in qualitative agreement with the previous predictions. We thus obtain a length reduction factor of about 3-5 as compared to the single-fiber design based on HNLF 1 and 2 .

Variations of the autocorrelation FWHM of the output pulses are also reported to illustrate the impact of the DCF length on the output pulse width variation over the input power operating range. The experimental measurements confirm the possibility to reduce the pulse duration fluctuations by using a longer intermediate segment, as predicted by numerical simulations (Fig. 6(c)). 

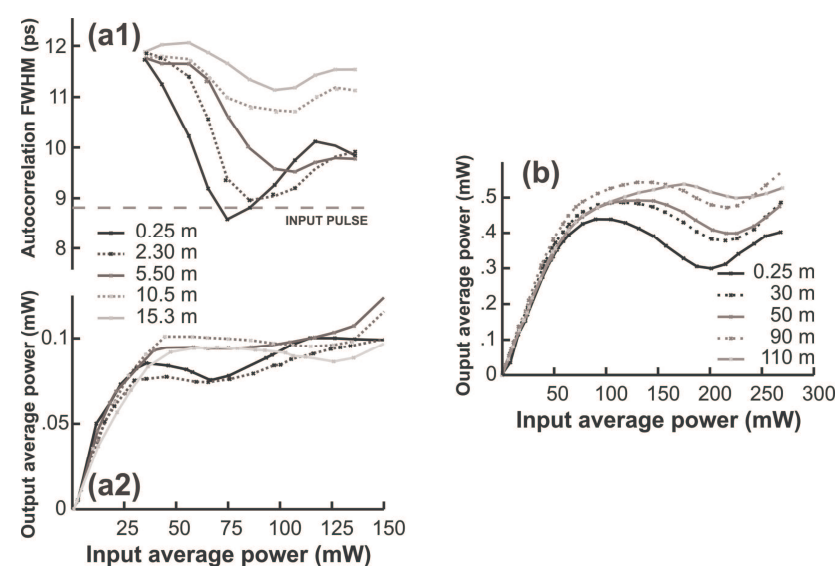

Fig. 9: Influence of the DCF length for different input powers: (a) Results for the 6.4 ps pulse train: output autocorrelation FWHM (a1) and TFs (a2), (b) TFs for the $10 \mathrm{GHz}$ setting for $\sim 20 \mathrm{ps}$-pulse train.

Finally, we experimentally demonstrate that by adjusting the operating power and length of DCF, pulses of substantially different temporal width (and consequently different repetition rates) can be straightforwardly accommodated. To preserve the benefit of the scaling rules [5], it is however necessary to keep the normalized filter frequency detuning $\Delta \lambda / \mathrm{R}$ constant (in our case 2.5). As a demonstration, we performed a second set of measurements using longer pulses of $\sim 20 \mathrm{ps}$ duration generated by an electro-absorption modulator-carved $\mathrm{CW}$ signal generated at a $10 \mathrm{GHz}$ repetition rate. In Fig. 9(b) we present the resulting TFs recorded after spectral filtering $(\Delta \lambda=25 \mathrm{GHz}$ and $\delta \mathrm{f}=20 \mathrm{GHz}$ ). Significant $\mathrm{TF}$ reshaping is observed and using $110 \mathrm{~m}$ of DCF between HNLF 1 and 2 allows a substantial flattening of the TF. The multi-segment configuration allows a length factor reduction of $\sim 40$ as compared to an idealized single fiber design [5].

\section{CONCLUSION}

We have demonstrated the various benefits obtained by implementing an original multi-segmented SPM-based regenerator that allows one to effectively separate the elements fulfilling the nonlinear and dispersive requirements of the device. These benefits include more compact and/or power efficient devices, increased design flexibility, improved versatility and straightforward device implementation/tuning using readily available lengths of commercial fibers. For this proof of principle demonstration we have used off-the-shelf fibers currently available in our laboratories however further reduction of the length of the highly nonlinear segments could be achieved by a more optimal choice of commercially available fibers. Furthermore, the DCF could be advantageously replaced by a chirped fiber Bragg grating designed to provide the normal linear dispersion required.

\section{ACKNOWLEDGEMENT}

The authors acknowledge the Furukawa Electric Company
(Japan) for the loan of one of highly nonlinear fiber used in this work.

\section{REFERENCES}

[1] N. J. Doran and D. Wood, "Nonlinear-optical loop miror," Opt. Lett., vol. 13 , pp. 56-58, 1988

[2] K. Suzuki, T. Tanemura, K. Taira, Y. Ozeki, and K. Kikuchi, "Alloptical regenerator using wavelength shift induced by cross-phase modulation in highly nonlinear dispersion-shifted fiber.," IEEE Photon. Technol. Lett., vol. 17, pp. 423-425, 2005.

[3] E. Ciaramella and S. Trillo, "All-optical signal reshaping via fourwave mixing in optical fibers.," IEEE Photon. Technol. Lett., vol. 12, p. $849,2000$.

[4] P. V. Mamyshev, "All-optical data regeneration based on self-phase modulation effect," in European Conference on Optical Communication, ECOC'98, Institute of Electrical and Electronics Engineering, Madrid, Spain, 1998, pp. 475-476.

[5] L. Provost, C. Finot, K. Mukasa, P. Petropoulos, and D. J. Richardson, "Design scaling rules for 2R-Optical Self-Phase Modulation-based regenerators 2R regeneration," Opt. Express, vol. 15, pp. 5100-5113, 2007.

[6] L. B. Fu, M. Rochette, V. G. Ta'eed, D. J. Moss, and B. J. Eggleton, "Investigation of self-phase modulation based optical regeneration in single mode As2Se3 chalcogenide glass fiber," Opt. Express, vol. 13, pp. 7639-7646, 2005 .

[7] P. P. Baveja, D. N. Maywar, and G. P. Agrawal, "Optimization of alloptical $2 \mathrm{R}$ regenerators operating at $40 \mathrm{~GB} / \mathrm{s}$ : role of dispersion," $J$. Lightw. Technol., 2009.

[8] L. Provost, C. Finot, K. Mukasa, P. Petropoulos, and D. J. Richardson, "Generalisation and experimental validation of design rules for self-phase modulation-based 2R-regenerators," in Optical Fiber Conference, OFC 2007, Anaheim, USA, 2007, p. OThB6.

[9] F. Parmigiani, S. Asimakis, N. Sugimoto, F. Koizumi, P. Petropoulos, and D. J. Richardson, "2R regenerator based on a 2-mlong highly nonlinear bismuth oxide fiber," Opt. Express, vol. 14, pp. 5038-5044, 2006.

[10] C. Finot, T. N. Nguyen, J. Fatome, T. Chartier, L. Bramerie, M. Gay, S. Pitois, and J. C. Simon, "Numerical study of an optical regenerator exploiting self-phase modulation and spectral offset filtering at 40 Gbit/s," Opt. Commun., vol. 281, pp. 2252-2264, 2008.

[11] M. Matsumoto, "Efficient all-optical 2R regeneration using selfphase modulation in bidirectional fiber configuration," Opt. Express, vol. 14, pp. 11018-11023, 2006.

[12] T.-H. Her, G. Raybon, and C. Headley, "Optimization of pulse regeneration at $40 \mathrm{~Gb} / \mathrm{s}$ based on spectral filtering of self-phase modulation in fiber," IEEE Photon. Technol. Lett., vol. 16, pp. 200$202,2004$.

[13] M. Matsuura and N. Kishi, "Wideband wavelength-flexible alloptical signal regeneration using gain-band tunable Raman amplification and self-phase-modulation-based spectral filtering," Opt. Lett., vol. 34, pp. 2420-2422, 2009.

[14] A. G. Striegler and B. Schmauss, "Analysis and optimization of SPM-based 2R signal regeneration at $40 \mathrm{~Gb} / \mathrm{s}$," J. Lightw. Technol., vol. 24, pp. 2835-2843, 2006.

[15] H. Murai, Y. Kanda, M. Kagawa, and S. Arahira, "Regenerative SPM-based wavelength conversion and field demonstration of 160Fb/s all-optical 3R operation," J. Lightw. Technol., 2009.

[16] T. I. Lakoba and M. Vasilyev, "A new robust regime for a dispersionmanaged multichannel 2R regenerator," Opt. Express, vol. 15, pp. 10061-10074, 2007.

[17] L. Provost, F. Parmigiani, C. Finot, K. Mukasa, P. Petropoulos, and D. J. Richardson, "Analysis of a two-channel $2 \mathrm{R}$ all-optical regenerator based on a counter-propagating configuration," Opt. Express, vol. 16, pp. 2264-2275, 2008.

[18] C. Kouloumentas, L. Provost, F. Parmigiani, S. Tsolakidis, P. Petropoulos, I. Tomkos, and D. J. Richardson, "Four-channel allfiber dispersion-managed 2R regenerator," IEEE Photon. Technol. Lett., vol. 20, pp. 1169-1171, 2008.

[19] W. J. Tomlinson, R. H. Stolen, and A. M. Johnson, "Optical wavebreaking of pulses in nonlinear optical fibers," Opt. Lett., vol. 10, pp. 457-459, 1985. 
[20] D. Anderson, M. Desaix, M. Lisak, and M. L. Quiroga-Teixeiro, "Wave-breaking in nonlinear optical fibers," J. Opt. Soc. Am. B, vol. 9, pp. 1358-1361, 1992.

[21] C. Finot, B. Kibler, L. Provost, and S. Wabnitz, "Beneficial impact of wave-breaking or coherent continuum formation in normally dispersive nonlinear fibers," J. Opt. Soc. Am. B, vol. 25, pp. 19381948, 2008.

[22] C. Finot, L. Provost, P. Petropoulos, and D. J. Richardson, "Parabolic pulse generation through passive nonlinear pulse reshaping in a normally dispersive two segment fiber device," Opt. Express, vol. 15, pp. 852-864, 2007.

[23] T. Hirooka and M. Nakazawa, "Parabolic pulse generation by use of a dispersion-decreasing fiber with normal group-velocity dispersion," Opt. Lett., vol. 29, pp. 498-500, 2004.

[24] L. Provost , C. Finot, P. Petropoulos, and D. J. Richardson, "2R Regeneration Architectures Based on Multi-Segmented Fibres," in European Conference on Optical Communications (ECOC'08) Brussels, Belgium, 2008, p. Th.1.B.1.

[25] B. Kibler, C. Billet, P. A. Lacourt, R. Ferrière, L. Larger, and J. M. Dudley, "Parabolic pulse generation in comb-like profiled dispersion decreasing fibre," Electron. Lett., vol. 42, pp. 965-966 2006.

[26] S. V. Chernikov, J. R. Taylor, and R. Kashyap, "Comblike dispersion-profiled fiber for soliton pulse train generation," Opt. Lett., vol. 19, pp. 539-541, 1994.

[27] T. Inoue, H. Tobioka, K. Igarashi, and S. Namiki, "Optical pulse compression based on stationary rescaled pulse propagation in a comblike profiled fiber," J. Lightw. Technol., vol. 24, pp. 2510-2522, 2006.

[28] G. P. Agrawal, Fiber-Optic Communication Systems: WileyInterscience, 2002. 